gr-qc/0305089

\title{
A new form of the C-metric
}

\author{
Kenneth Hong and Edward Teo \\ Department of Physics, National University of Singapore, Singapore 119260
}

\begin{abstract}
The usual form of the C-metric has the structure function $G(\xi)=1-\xi^{2}-2 m A \xi^{3}$, whose cubic nature can make calculations cumbersome, especially when explicit expressions for its roots are required. In this paper, we propose a new form of the C-metric, with the explicitly factorisable structure function $G(\xi)=\left(1-\xi^{2}\right)(1+2 m A \xi)$. Although this form is related to the usual one by a coordinate transformation, it has the advantage that its roots are now trivial to write down. We show that this leads to potential simplifications, for example, when casting the C-metric in Weyl coordinates. These results also extend to the charged C-metric, whose structure function can be written in the new form $G(\xi)=\left(1-\xi^{2}\right)\left(1+r_{+} A \xi\right)\left(1+r_{-} A \xi\right)$, where $r_{ \pm}$are the usual locations of the horizons in the Reissner-Nordström solution. As a by-product, we explicitly cast the extremally charged C-metric in Weyl coordinates.
\end{abstract}




\section{Introduction}

The C-metric is well known to describe a pair of black holes undergoing uniform acceleration. It is usually written in a form first used by Kinnersley and Walker [1]:

$$
\mathrm{d} s^{2}=\frac{1}{A^{2}(x-y)^{2}}\left[G(y) \mathrm{d} t^{2}-\frac{\mathrm{d} y^{2}}{G(y)}+\frac{\mathrm{d} x^{2}}{G(x)}+G(x) \mathrm{d} \phi^{2}\right],
$$

where the structure function $G$ is given by

$$
G(\xi)=1-\xi^{2}-2 m A \xi^{3}
$$

Here, $m$ and $A$ are positive parameters related to the mass and acceleration of the black hole, satisfying $m A<1 / \sqrt{27}$. The fact that $G$ is a cubic polynomial in $\xi$ means one in general cannot write down simple expressions for its roots. Since these roots play an important role in almost every analysis of the C-metric, most results have to be expressed implicitly in terms of them. Any calculation which requires their explicit forms would naturally be very tedious if not impossible to carry out (see, e.g., [2, 3, 4]).

In this paper, we advocate a new form of the C-metric, given by (11) but with the structure function

$$
G(\xi)=\left(1-\xi^{2}\right)(1+2 m A \xi)
$$

where now $m A<1 / 2$. When expanded, it formally differs from (2) only in the presence of a new linear term. However, recall that the coefficient of the linear term (and also that of the quadratic term) is a purely kinematical parameter [5] and so changing it will not alter the physical interpretation of the spacetime. Indeed, the two forms of the C-metric are related by a coordinate transformation together with a redefinition of $m$ and $A$. But with the new structure function (3), it is a trivial matter to write down its roots explicitly, and this would in turn simplify certain analyses of the C-metric.

One important example of such a simplification is when the C-metric is written in Weyl coordinates 6, 3, 7, 8]. Recall that the $z$-axis in these coordinates consists of a rod of finite length representing the black hole event horizon, together with a semi-infinite rod representing the acceleration horizon. In the usual treatment, the positions of the rod ends are given as roots of a cubic polynomial and are therefore very complicated in general. However, we will show that the new form of the C-metric leads to a corresponding rod structure that is more elegant than the usual one, in the sense that the positions of the rod ends are given by very simple and natural expressions. 
It turns out that the aforementioned results also extend almost directly to the charged C-metric. Therefore, in the interest of generality, we shall present only the results for the charged C-metric in this paper, with the standard C-metric being understood to arise as a special case. In Sec. 2, the coordinate transformation relating the old and new forms of the charged C-metric is presented. In Sec. 3, a few of its basic properties are discussed with reference to the new form. The transformation to Weyl coordinates is then covered in Sec. 4, as an example of the advantages of using the new form of the C-metric. The paper ends with a discussion of how these results can be extended to other related spacetime solutions.

\section{Coordinate transformation}

In its usual form, the charged C-metric is given by the line element and electromagnetic potential [1]:

$$
\mathrm{d} s^{2}=\frac{1}{\tilde{A}^{2}(\tilde{x}-\tilde{y})^{2}}\left[\tilde{G}(\tilde{y}) \mathrm{d} \tilde{t}^{2}-\frac{\mathrm{d} \tilde{y}^{2}}{\tilde{G}(\tilde{y})}+\frac{\mathrm{d} \tilde{x}^{2}}{\tilde{G}(\tilde{x})}+\tilde{G}(\tilde{x}) \mathrm{d} \tilde{\phi}^{2}\right], \quad \mathcal{A}=\tilde{q} \tilde{y} \mathrm{~d} \tilde{t}
$$

with

$$
\tilde{G}(\tilde{\xi})=1-\tilde{\xi}^{2}-2 \tilde{m} \tilde{A} \tilde{\xi}^{3}-\tilde{q}^{2} \tilde{A}^{2} \tilde{\xi}^{4}
$$

It clearly reduces to (11), (2) when the electric charge parameter $\tilde{q}$ vanishes. Here, we have introduced tildes on the top of the coordinates and parameters of this solution, to distinguish them from those that would appear in the new form of the C-metric. As in the uncharged case, we would like the latter to have a factorisable structure function as follows:

$$
\mathrm{d} s^{2}=\frac{1}{A^{2}(x-y)^{2}}\left[G(y) \mathrm{d} t^{2}-\frac{\mathrm{d} y^{2}}{G(y)}+\frac{\mathrm{d} x^{2}}{G(x)}+G(x) \mathrm{d} \phi^{2}\right], \quad \mathcal{A}=q y \mathrm{~d} t
$$

with

$$
G(\xi)=\left(1-\xi^{2}\right)\left(1+r_{+} A \xi\right)\left(1+r_{-} A \xi\right)
$$

Here, we have introduced $r_{ \pm}$that are related to the new mass and charge parameters $m$ and $q$ by

$$
r_{ \pm} \equiv m \pm \sqrt{m^{2}-q^{2}}
$$

Moreover we assume that

$$
0 \leq r_{-} A \leq r_{+} A<1
$$


so that $G(\xi)$ has four distinct real roots, except in the uncharged case $q=0$ and the extremally charged case $|q|=m$, when it has three distinct roots.

To turn the C-metric (4), (15) into the form (6), (17), consider the following coordinate transformation:

$$
\begin{aligned}
\tilde{x} & =B c_{0}\left(x-c_{1}\right), \\
\tilde{y} & =B c_{0}\left(y-c_{1}\right), \\
\tilde{t} & =\frac{c_{0}}{B} t, \\
\tilde{\phi} & =\frac{c_{0}}{B} \phi,
\end{aligned}
$$

where $c_{0}, c_{1}$ and $B$ are real constants. It immediately follows that we must require

$$
\begin{aligned}
\tilde{A} & =\frac{1}{B} A, \\
\tilde{G}(\tilde{\xi}) & =B^{2} G(\xi),
\end{aligned}
$$

in order to preserve the form of the line element. Equating the five independent coefficients in (11b) for either $\xi=x$ or $y$, we have

$$
\begin{aligned}
\tilde{q} & =\frac{q}{c_{0}^{2}}, \\
\tilde{m} & =\frac{1}{c_{0}^{3}}\left(m+2 q^{2} c_{1} A\right), \\
c_{0}^{2} & =1-q^{2} A^{2}+6 m A c_{1}+6 q^{2} A^{2} c_{1}^{2}, \\
\frac{1}{B^{2}} & =1+\left(1-q^{2} A^{2}\right) c_{1}^{2}+4 m A c_{1}^{3}+3 q^{2} A^{2} c_{1}^{4},
\end{aligned}
$$

and

$$
4 q^{2} A^{2} c_{1}^{3}+6 m A c_{1}^{2}+2\left(1-q^{2} A^{2}\right) c_{1}-2 m A=0 .
$$

Observe that (12e) is a cubic equation in $c_{1}$ with a non-positive discriminant, so it has three real roots. Furthermore, the product of these three roots has to equal $m /\left(2 q^{2} A\right)>0$, which implies that at least one of them is positive. Choosing a positive solution for $c_{1}$, one can in principle deduce the other two constants using (12c) and (12d). It can be seen, using the positivity of $c_{1}$ and the condition (9), that the right-hand sides of these two equations are positive, thus ensuring that $c_{0}$ and $B$ are real. With these three constants at hand, one can then obtain the relationships between the tilded and non-tilded quantities using the remaining equations of (12), (11a) and (10). Note also that these transformations correctly turn the electromagnetic potential in (4) into the one in (6), up to an irrelevant additive constant. 
The explicit expression for $c_{1}$ is, of course, very complicated in general. In a certain sense, the difficulty in finding the roots of $\tilde{G}(\tilde{\xi})$ in the original coordinates has been shifted to determining $c_{1}$ instead. However, unless we are trying to transform a quantity calculated in the original coordinates to the new coordinates, the explicit form of $c_{1}$ is not required. In the rest of this paper, we shall use the new form of the C-metric (6), (77) as the starting point of our analysis.

In closing this section, we remark that Dowker et al. [9] have in fact considered a form of the charged C-metric with the structure function

$$
\tilde{G}(\tilde{\xi})=\left(1-\tilde{\xi}^{2}-\tilde{r}_{+} \tilde{A} \tilde{\xi}^{3}\right)\left(1+\tilde{r}_{-} \tilde{A} \tilde{\xi}\right) .
$$

It is in some sense an intermediate form between Kinnersley and Walker's structure function (5) and ours (77), and was the inspiration for the latter. It can be checked that a transformation similar to (10) and (11) will bring this form of the charged C-metric into our form. We also mention that a certain non-extremal $\mathrm{U}(1)^{n}$-charged C-metric solution was found in a factorised form similar to ours in [10, but its implications were not explored.

\section{Properties}

The properties of the C-metric in the new form (6), (17) can be analysed almost in parallel with those in the old form. For this reason, we will not repeat everything that is known about it here, but only discuss a few more important properties.

Let us denote the four real roots of (17) by

$$
\xi_{1} \equiv-\frac{1}{r_{-} A}, \quad \xi_{2} \equiv-\frac{1}{r_{+} A}, \quad \xi_{3} \equiv-1, \quad \xi_{4} \equiv 1
$$

which obey $\xi_{1} \leq \xi_{2}<\xi_{3}<\xi_{4}$. In order to have the correct spacetime signature, we assume the $x$ and $y$ coordinates take the ranges $\xi_{3} \leq x \leq \xi_{4}$ and $\xi_{2} \leq y \leq \xi_{3}$, respectively. As in the usual case, asymptotic infinity is located at $x=y=\xi_{3}$. The black hole event horizon is located at $y=\xi_{2}$, while the acceleration horizon is at $y=\xi_{3}$. The line $x=\xi_{4}$ is the part of the symmetry axis between the event and acceleration horizons, while $x=\xi_{3}$ is that part of the symmetry axis joining up the event horizon with asymptotic infinity.

It turns out that there are in general conical singularities along $x=\xi_{3}$ and $\xi_{4}$. If we 
take the angle $\phi$ to have period $\Delta \phi$, then the deficit angle along $x=\xi_{i}$ is

$$
\delta=2 \pi-\left|\frac{\Delta \phi \mathrm{d} \sqrt{g_{\phi \phi}}}{\sqrt{g_{x x}} \mathrm{~d} x}\right|_{x=\xi_{i}}=2 \pi-\alpha_{i} \Delta \phi,
$$

where $\alpha_{i} \equiv \frac{1}{2}\left|G^{\prime}\left(\xi_{i}\right)\right|$. It can be checked that

$$
\alpha_{3}=1-2 m A+q^{2} A^{2}, \quad \alpha_{4}=1+2 m A+q^{2} A^{2},
$$

which are considerably simpler than the corresponding expressions in the usual case when $G(\xi)$ is given by (51). However, the physics of these conical singularities remains unchanged. Note that both conical singularities cannot be made to vanish at the same time. If we choose to remove the conical deficit along $x=\xi_{4}$ with the choice $\Delta \phi=\frac{2 \pi}{\alpha_{4}}$, then there is a positive deficit angle along $x=\xi_{3}$. This can be interpreted as a semi-infinite cosmic string pulling on the black hole. Alternatively, we can choose to remove the conical deficit along $x=\xi_{3}$ with the choice $\Delta \phi=\frac{2 \pi}{\alpha_{3}}$, resulting in a negative deficit angle along $x=\xi_{4}$. This can be interpreted as a strut pushing on the black hole. The strut continues past the acceleration horizon, and joins up with a 'mirror' black hole on the other side of it.

If we perform the coordinate transformation

$$
t=A t^{\prime}, \quad x=\cos \theta, \quad y=-\frac{1}{A r},
$$

and take the limit $A \rightarrow 0$, the solution (6) reduces to

$$
\begin{aligned}
\mathrm{d} s^{2} & =-\left(1-\frac{r_{+}}{r}\right)\left(1-\frac{r_{-}}{r}\right) \mathrm{d} t^{\prime 2}+\frac{\mathrm{d} r^{2}}{\left(1-\frac{r_{+}}{r}\right)\left(1-\frac{r_{-}}{r}\right)}+r^{2}\left(\mathrm{~d} \theta^{2}+\sin ^{2} \theta \mathrm{d} \phi^{2}\right), \\
\mathcal{A} & =-\frac{q}{r} \mathrm{~d} t^{\prime},
\end{aligned}
$$

which is the Reissner-Nordström solution describing a single static charged black hole, with horizons located at $r=r_{ \pm}$. (17) is the same transformation as in the usual case, and shows that the parameter $A$, like $\tilde{A}$, governs the acceleration of the black hole. On the other hand, $m$ and $q$, like $\tilde{m}$ and $\tilde{q}$, are respectively the ADM mass and charge of the black hole in this limit. It is only in the case of non-zero acceleration that $m$ and $q$ differ from their usual counterparts $\tilde{m}$ and $\tilde{q}$, in a way that can be deduced from (12a) and (12b). In this sense, the new parameters $m$ and $q$ have the advantage of not being 'dressed' by $A$, and retain their original interpretations given by the 'bare' $A=0$ case. 


\section{Weyl form}

In this section, we will cast the C-metric (6), (17) in the Weyl form

$$
\mathrm{d} s^{2}=-\mathrm{e}^{2 \lambda} \mathrm{d} t^{2}+\mathrm{e}^{2(\nu-\lambda)}\left(\mathrm{d} \rho^{2}+\mathrm{d} z^{2}\right)+\mathrm{e}^{-2 \lambda} \rho^{2} \mathrm{~d} \phi^{2}
$$

(where $\lambda$ and $\nu$ are functions of $\rho$ and $z$ only) and analyse its rod structure. The treatment will follow [3, 4, 7, 11, 8, 12, although there will be some crucial simplifications which we will highlight.

To begin, consider the coordinate transformation:

$$
\rho=\frac{\sqrt{-G(x) G(y)}}{A^{2}(x-y)^{2}}, \quad z=\frac{(1-x y)\left[1+m A(x+y)+q^{2} A^{2} x y\right]}{A^{2}(x-y)^{2}}
$$

The Weyl metric functions in terms of $x$ and $y$ are

$$
\mathrm{e}^{2 \lambda}=\frac{-G(y)}{A^{2}(x-y)^{2}}, \quad \mathrm{e}^{2 \nu}=\frac{1}{A^{4}(x-y)^{4}}\left[\left(\frac{\partial \rho}{\partial y}\right)^{2}+\left(\frac{\partial z}{\partial y}\right)^{2}\right]^{-1}
$$

In order to express these metric functions in terms of $\rho$ and $z$, we need to invert the relations (20). Following the procedure in [8, 12, we would like to find constants $\alpha_{i}$ and $z_{i}(i=1,2,3)$, such that

$$
\rho^{2}+\left(z-z_{i}\right)^{2}=\left[\frac{\alpha_{1}+\alpha_{2}(x+y)+\alpha_{3} x y}{A^{2}(x-y)}\right]^{2} .
$$

Multiplying this equation by $A^{4}(x-y)^{2}$, and using (20), we may compare the coefficients on both sides to obtain

$$
\begin{aligned}
& \alpha_{1}^{2}=A^{2}\left(m^{2}-q^{2}\right)+1-2 A^{2} z_{i}, \\
& \alpha_{2}^{2}=A^{2}\left(q^{2}+A^{2} z_{i}^{2}\right), \\
& \alpha_{3}^{2}=A^{2}\left[\left(m^{2}-q^{2}\right)+q^{2} A^{2}\left(q^{2}+2 z_{i}\right)\right],
\end{aligned}
$$

where $z_{i}$ are the roots of the cubic equation

$$
\left(2 A^{2} z_{i}-1+q^{2} A^{2}\right)\left(A^{2} z_{i}^{2}-m^{2}+q^{2}\right)=0 .
$$

Unlike the corresponding equation in the usual treatment [11, this equation is explicitly factorisable, and the roots are simply

$$
z_{1}=-z_{2}=-\frac{\sqrt{m^{2}-q^{2}}}{A}, \quad z_{3}=\frac{1-q^{2} A^{2}}{2 A^{2}} .
$$


It can be checked that $z_{1} \leq z_{2}<z_{3}$.

Now, it turns out that $z_{i}$ play a very important role in the rod structure of the C-metric. Using the mapping (201), it can be seen that the black hole event horizon is located on the $z$-axis between $z_{1}$ and $z_{2}$, while the acceleration horizon stretches from $z_{3}$ to infinity. The corresponding rod structure is sketched in Fig. 1.

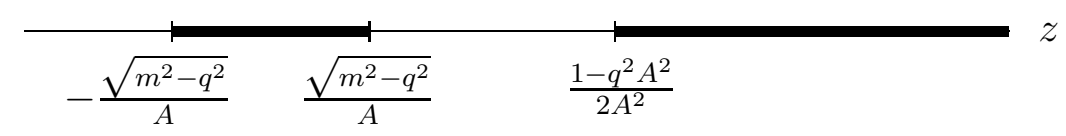

Figure 1: The position of the rods along the $z$-axis, in the new coordinates.

Note that the finite rod representing the event horizon is centered at $z=0$, with length
$\frac{2 \sqrt{m^{2}-q^{2}}}{A}$. Up to a scale factor of $\frac{1}{A}$, this is exactly the same as the length of the rod in the Weyl form of the Reissner-Nordström solution (see, e.g., [13]). This is in contrast to the usual treatment of the C-metric in Weyl coordinates where $z_{1}$ and $z_{2}$ depend on $A$ in a non-trivial manner [11, which would result in the finite rod changing length as well as shifting along the $z$-axis as $A$ is increased.

To find $x$ and $y$ explicitly in terms of $\rho$ and $z$, it is convenient to introduce functions $R_{i}$ defined by

$$
R_{i} \equiv \sqrt{\rho^{2}+\left(z-z_{i}\right)^{2}} .
$$

After substituting (23), (25) into (22) and taking square roots, we have

$$
\begin{aligned}
& R_{1}=\frac{1+A \sqrt{m^{2}-q^{2}}+m A(x+y)-A\left(\sqrt{m^{2}-q^{2}}-q^{2} A\right) x y}{A^{2}(x-y)}, \\
& R_{2}=\frac{1-A \sqrt{m^{2}-q^{2}}+m A(x+y)+A\left(\sqrt{m^{2}-q^{2}}+q^{2} A\right) x y}{A^{2}(x-y)}, \\
& R_{3}=-\frac{2 m A+\left(1+q^{2} A^{2}\right)(x+y)+2 m A x y}{2 A^{2}(x-y)},
\end{aligned}
$$

where we have chosen the signs of $\alpha_{i}$, as well as the overall signs of $R_{i}$, appropriate for the C-metric [7, 11, 8]. (27a-c) are three dependent equations for $x$ and $y$ as functions of $\rho$ and $z$, and we may solve them to obtain

$$
x=\frac{F_{1}+F_{2}}{2 F_{0}}, \quad y=\frac{F_{1}-F_{2}}{2 F_{0}},
$$


where

$$
\begin{aligned}
& F_{0} \equiv \sqrt{m^{2}-q^{2}}\left[\left(1+q^{2} A^{2}\right)\left(R_{1}+R_{2}\right)+4 m A R_{3}\right]-A\left(2 m^{2}-q^{2}-q^{4} A^{2}\right)\left(R_{1}-R_{2}\right), \\
& F_{1} \equiv-4 \sqrt{m^{2}-q^{2}}\left[m A\left(R_{1}+R_{2}\right)+\left(1+q^{2} A^{2}\right) R_{3}\right]+2 m\left(1-q^{2} A^{2}\right)\left(R_{1}-R_{2}\right) \\
& F_{2} \equiv \frac{2}{A^{2}} \sqrt{m^{2}-q^{2}}\left(1-2 m A+q^{2} A^{2}\right)\left(1+2 m A+q^{2} A^{2}\right)
\end{aligned}
$$

This would allow us to cast the metric functions (21) in terms of $\rho$ and $z$. Although the expressions for $F_{0}, F_{1}$ and $F_{2}$ are much more manageable than the usual ones [8], they can still give rather complicated expressions for the metric functions.

For simplicity, let us now specialise to the case of an extremally charged accelerating black hole, i.e., $|q|=m$. In this case, the finite rod shrinks down to a point at the origin:

$$
z_{1}=z_{2}=0, \quad z_{3}=\frac{1-m^{2} A^{2}}{2 A^{2}}
$$

Hence, $(27 \mathrm{a}-\mathrm{c})$ become

$$
\begin{aligned}
& R_{1}=R_{2}=\frac{1+m A(x+y)+m^{2} A^{2} x y}{A^{2}(x-y)} \\
& R_{3}=-\frac{2 m A+\left(1+m^{2} A^{2}\right)(x+y)+2 m A x y}{2 A^{2}(x-y)} .
\end{aligned}
$$

Solving for $x$ and $y$, we have

$$
\begin{aligned}
& x=-\frac{m A R_{1}+R_{3}-z_{3}}{R_{1}+m A\left(R_{3}-z_{3}\right)}, \\
& y=-\frac{m A R_{1}+R_{3}+z_{3}}{R_{1}+m A\left(R_{3}+z_{3}\right)} .
\end{aligned}
$$

Finally, substituting (32) into the Weyl metric functions (21),

$$
\begin{aligned}
\mathrm{e}^{2 \lambda} & =\left[R_{3}-\left(z-z_{3}\right)\right]\left[\frac{R_{1}+m A\left(R_{3}-z_{3}\right)}{R_{1}+m A\left(R_{3}+z_{3}\right)}\right]^{2}, \\
\mathrm{e}^{2(\nu-\lambda)} & =\frac{\left[R_{1}+m A\left(R_{3}+z_{3}\right)\right]^{2}\left[R_{1}+m A\left(R_{3}-z_{3}\right)\right]^{2}}{32\left(z_{3} A^{2} R_{1}\right)^{4} R_{3}} .
\end{aligned}
$$

The electromagnetic potential $\mathcal{A}=m y \mathrm{~d} t$ can also be readily read off using (32b). To our knowledge, this is the first time that the charged C-metric (extremal or otherwise) has been cast in Weyl form explicitly. It would be interesting to see if (33) can be generalised to a Weyl solution describing multiple extremal black holes undergoing uniform acceleration. 


\section{Discussion}

In this paper, we have shown how a new form of the C-metric offers advantages over the traditional form. With a factorisable structure function, its roots can be explicitly read off from it, leading to simplifications in the analysis of the C-metric. In particular, we demonstrated how its rod structure in Weyl coordinates simplifies when the new form is used.

These results would also apply to spacetimes that are closely related to the charged C-metric, such as the dilatonic C-metric [9]. Another example is the rotating black ring solution in five dimensions [14, which can be obtained by analytic continuation of the Cmetric solution in Kaluza-Klein theory 9]. Using a transformation similar to (10) together with a suitable redefinition of parameters, it can be written in the form

$$
\begin{aligned}
\mathrm{d} s^{2}= & -\frac{F(x)}{F(y)}\left(\mathrm{d} t+\sqrt{\frac{\nu}{\xi_{1}}} \frac{\xi_{2}-y}{A} \mathrm{~d} \psi\right)^{2} \\
& +\frac{1}{A^{2}(x-y)^{2}}\left[-F(x)\left(G(y) \mathrm{d} \psi^{2}+\frac{F(y)}{G(y)} \mathrm{d} y^{2}\right)+F(y)^{2}\left(\frac{\mathrm{d} x^{2}}{G(x)}+\frac{G(x)}{F(x)} \mathrm{d} \phi^{2}\right)\right],
\end{aligned}
$$

with the explicitly factorisable structure functions

$$
F(\xi)=1-\xi / \xi_{1}, \quad G(\xi)=\left(1-\xi^{2}\right)(1-\nu \xi)
$$

The roots of $G(\xi)$ are now given by $\xi_{2}=-1, \xi_{3}=1, \xi_{4}=\frac{1}{\nu}$ (following the labelling of [14]), where $0<\nu<1$. For $\xi_{1}=\frac{1+\nu^{2}}{2 \nu}$, we have a regular solution free of conical singularities.

Besides simplifying known results, the new form of the C-metric opens up the possibility of performing calculations that were previously impractical if not impossible. One example that was presented in this paper is the calculation of the explicit form of the extremal charged C-metric in Weyl coordinates.

Another example is the possibility of starting with the Weyl form of a certain spacetime solution, and working backwards to cast it in C-metric-type coordinates. This is in principle possible for any solution whose rod structure contains exactly three 'characteristic points' $z_{i}$ $(i=1,2,3)$ where the rods terminate.* However, in the old formalism, the positions of these three points have to obey a cubic equation of the form

$$
2 A^{4} z_{i}^{3}-A^{2} z_{i}^{2}+m^{2}=0 .
$$

${ }^{*}$ This is analogous to the well-known result that any Weyl solution with two characteristic points $\pm z_{0}$ can be simplified by casting it in prolate spheroidal coordinates $(p, q)$ defined by $p \equiv \frac{1}{2 z_{0}}\left(R_{+}+R_{-}\right)$and $q \equiv \frac{1}{2 z_{0}}\left(R_{+}-R_{-}\right)$, where $R_{ \pm} \equiv \sqrt{\rho^{2}+\left(z \pm z_{0}\right)^{2}}$. 
This makes it cumbersome to relate $z_{i}$ to the new parameters $m$ and $A$ that would appear in the final form of the solution in C-metric-type coordinates. On the other hand, when using the new formalism, the three characteristic points have to satisfy

$$
\left(2 A^{2} z_{i}-1\right)\left(A^{2} z_{i}^{2}-m^{2}\right)=0 .
$$

It is now a trivial matter to relate $z_{i}$ to $m$ and $A$. As an application of this method, a Weyl solution describing a black dihole (i.e., a pair of oppositely charged extremal black holes) in five dimensions was transformed to C-metric-type coordinates in [15], simplifying the solution and thus making its properties more amenable to analysis. This method can also be applied to the multiple accelerating black hole solution [16], if one wants to close in to a single accelerating black hole and cast it in C-metric-type coordinates.

A natural question at this stage is whether the results of this paper would apply to other generalisations of the C-metric, such as the de Sitter / anti-de Sitter C-metric [5, 17, 18, 19] or the rotating C-metric [5, 12, 20]. In the latter case, while it is straightforward to write down a form for it which has a naturally factorisable structure function, this form cannot be obtained from the usual form of the rotating C-metric by a coordinate transformation. This leads to the question of what is the physical difference between these two solutions. Work in this direction is in progress.

\section{References}

[1] W. Kinnersley and M. Walker, "Uniformly accelerating charged mass in general relativity," Phys. Rev. D 2 (1970) 1359.

[2] H. Farhoosh and R. L. Zimmerman, "Killing horizons and dragging of the inertial frame about a uniformly accelerating particle," Phys. Rev. D 21 (1980) 317.

[3] W. B. Bonnor, "The sources of the vacuum C-metric," Gen. Rel. Grav. 15 (1983) 535.

[4] W. B. Bonnor, "The C-metric with $m=0, e \neq 0$," Gen. Rel. Grav. 16 (1984) 269.

[5] J. F. Plebanski and M. Demianski, "Rotating, charged, and uniformly accelerating mass in general relativity," Annals Phys. 98 (1976) 98.

[6] B. B. Godfrey, "Horizons in Weyl metrics exhibiting extra symmetries," Gen. Rel. Grav. 3 (1972) 3. 
[7] F. H. J. Cornish and W. J. Uttley, "The interpretation of the C metric: the vacuum case," Gen. Rel. Grav. 27 (1995) 439.

[8] V. Pravda and A. Pravdová, "Boost-rotation symmetric spacetimes - review," Czech. J. Phys. 50 (2000) 333 arXiv:gr-qc/0003067.

[9] F. Dowker, J. P. Gauntlett, D. A. Kastor and J. Traschen, "Pair creation of dilaton black holes," Phys. Rev. D 49 (1994) 2909 arXiv:hep-th/9309075.

[10] R. Emparan, "Composite black holes in external fields," Nucl. Phys. B 490 (1997) 365 arXiv:hep-th/9610170.

[11] F. H. J. Cornish and W. J. Uttley, "The interpretation of the C metric: the charged case when $e^{2} \leq m^{2}, "$ Gen. Rel. Grav. 27 (1995) 735.

[12] J. Bičák and V. Pravda, "Spinning C-metric: radiative spacetime with accelerating, rotating black holes," Phys. Rev. D 60 (1999) 044004 arXiv:gr-qc/9902075.

[13] R. Emparan and E. Teo, "Macroscopic and microscopic description of black diholes," Nucl. Phys. B 610 (2001) 190 arXiv:hep-th/0104206.

[14] R. Emparan and H. S. Reall, "A rotating black ring in five dimensions," Phys. Rev. Lett. 88 (2002) 101101 arXiv:hep-th/0110260.

[15] E. Teo, "Black diholes in five dimensions," (in preparation).

[16] H. F. Dowker and S. N. Thambyahpillai, "Many accelerating black holes," Class. Quant. Grav. 20 (2003) 127 arXiv:gr-qc/0105044.

[17] J. Podolský and J. B. Griffiths, "Uniformly accelerating black holes in a de Sitter universe," Phys. Rev. D 63 (2001) 024006 arXiv:gr-qc/0010109.

[18] Ó. J. C. Dias and J. P. S. Lemos, "Pair of accelerated black holes in a de Sitter background: the dS C-metric," Phys. Rev. D 67 (2003) 084018 arXiv:hep-th/0301046.

[19] Ó. J. C. Dias and J. P. S. Lemos, "Pair of accelerated black holes in an anti-de Sitter background: the AdS C-metric," Phys. Rev. D 67 (2003) 064001 arXiv:hep-th/0210065.

[20] P. S. Letelier and S. R. Oliveira, "Uniformly accelerated black holes," Phys. Rev. D 64 (2001) 064005 arXiv:gr-qc/9809089. 